\title{
Eccentric Regurgitant Jet Indicator
}

National Cancer Institute

\section{Source}

National Cancer Institute. Eccentric Regurgitant Jet Indicator. NCI Thesaurus. Code C139057.

An indication as to whether an eccentric regurgitant jet direction is present. 